\title{
Plug-and-Play Supervisory Control Using Muscle and Brain Signals for Real-Time Gesture and Error Detection
}

\author{
Joseph DelPreto*, Andres F. Salazar-Gomez*, Stephanie Gil*, Ramin M. Hasani ${ }^{\dagger}$, \\ Frank H. Guenther ${ }^{\ddagger}$, and Daniela Rus*
}

\begin{abstract}
Control of robots in safety-critical tasks and situations where costly errors may occur is paramount for realizing the vision of pervasive human-robot collaborations. For these cases, the ability to use human cognition in the loop can be key for recuperating safe robot operation. This paper combines two streams of human biosignals, electrical muscle and brain activity via EMG and EEG, respectively, to achieve fast and accurate human intervention in a supervisory control task. In particular, this paper presents an end-to-end system for continuous rollingwindow classification of gestures that allows the human to actively correct the robot on demand, discrete classification of Error-Related Potential signals (unconsciously produced by the human supervisor's brain when observing a robot error), and a framework that integrates these two classification streams for fast and effective human intervention. The system also allows "plug-and-play" operation, demonstrating accurate performance even with new users whose biosignals have not been used for training the classifiers. The resulting hybrid control system for safety-critical situations is evaluated with 7 untrained human subjects in a supervisory control scenario where an autonomous robot performs a multi-target selection task.
\end{abstract}

\section{INTRODUCTION}

As robots become more prevalent in homes, factory floors, and other safety-critical settings, detecting and correcting robot errors becomes increasingly important. In such situations, errors can be costly and difficult to remedy due to potential hardware damage or safety risks. A fast, reliable, and intuitive framework for supervisory control of robots is therefore required. If a robot could be taught to detect nonverbal human cues such as distress signals and hand gestures as reliably as a collaborating human partner, then interactions with robots would become more efficient and supervision or collaboration would become more effective.

Ideally, communication between the human and robot would be intuitive and have low latency. Using human biosignals such as muscle or brain activity via Electromyography (EMG) or Electroencephalography (EEG), respectively, has become a promising technique for fast, natural, and seamless humanrobot interaction (HRI). EMG interfaces can be used for controlling dynamical systems such as prosthetic limbs, while EEG signals can be used to detect higher-level cognitive states using signals such as the Error-Related Potential (ErrP), which is evoked upon perception of an error.

* MIT Distributed Robotics Lab, Cambridge, MA 02139

\{delpreto, salacho, sgil, rus\}@csail.mit.edu

†Vienna University of Technology Cyber-Physical Systems Group, Austria ramin.hasani@tuwien.ac.at

‡Boston University Guenther Lab, Boston, MA 02215

guenther@bu.edu

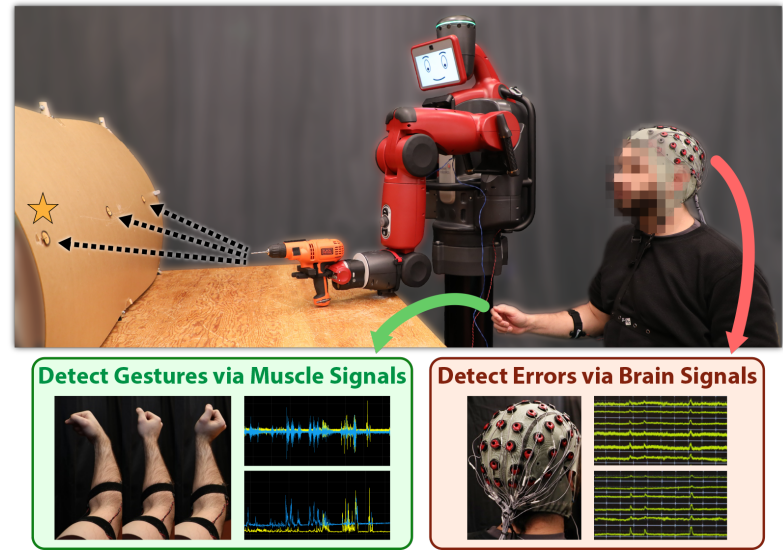

Fig. 1: A user supervises and controls an autonomous robot using brain signals to detect mistakes and muscle signals to select targets. ${ }^{1}$

Despite their potential, both biosignals have significant shortcomings as reliable sources of fast human-robot communication. EEG signals are noisy, making reliable real-time ErrP detection challenging. EMG signals are generally easier to detect and can provide a higher degree of control beyond binary choice, but they are also noisy and often difficult to map to motions. Both types of signals vary greatly over time and across subjects, leading to the common practice of a training phase for tuning the classifiers. This practice, however, precludes a "plug-and-play" system where new users can begin controlling the robot from their first interaction.

By combining EEG and EMG systems, this paper capitalizes on the human's cognitive ability to judge whether a robot made a mistake and their physical ability to indicate correct actions once a mistake has been made. The unconscious ErrP signal does not require user training, leading to fast passive error detection relying only on attention, while gestures provide an active means of reliably indicating desired behavior. The resulting "plug-and-play" hybrid control framework enables high performance in a supervisory control scenario where a robot conducts a multi-target selection task for a mock drilling operation. A human supervisor observes the autonomous robot and mentally judges whether it chose the correct target. If an ErrP or a gesture is detected, the robot halts and requests assistance. The human then gestures to the left or right to naturally scroll through possible targets. Once the correct target is selected, the robot resumes autonomous operation. This combination of EEG and EMG thus leads to fast passive detection and reliable active correction of mistakes.

\footnotetext{
${ }^{1}$ Videos are available at http://people.csail.mit.edu/delpreto/rss2018
} 
To achieve this, two independent classification pipelines process EEG and EMG signals in real time. The EEG pipeline evaluates the combined output of two neural networks on a buffer of time-locked EEG signals, outputting whether an ErrP is detected upon robot target selection. The EMG pipeline continuously classifies surface EMG signals on a rolling basis, evaluating a neural network 80 times per second to output whether a left gesture, a right gesture, or no gesture is detected. This continuous classification allows the user to exert active control over the robot at any point during operation. In addition, a new user does not need to provide training data for the EEG or EMG classifiers; training is done on a corpus of data from previous users, allowing the interface to be "plug-and-play" and broadening its applicability to untrained or novice users.

Paper contributions: This paper focuses on effective and reliable supervisory control in HRI tasks. In particular, its contributions are as follows:

- A framework for combining error detection via EEG with gesture detection via EMG to enable fast, reliable, and intuitive supervisory control of autonomous robots;

- A signal-processing and classification pipeline for continuously detecting gestures based on surface EMG signals, without requiring training on current user data;

- A classification pipeline for detecting ErrPs in EEG signals, without requiring training on current user data;

- An end-to-end "plug-and-play" system for supervising and controlling a robot during a target selection task;

- Experimental results from 7 untrained subjects, demonstrating effective supervision of a mock drilling operation where the robot chooses from 3 possible targets.

\section{RELATED WORK}

This paper builds upon bodies of work investigating humanrobot interaction and classification of human biosignals.

\section{A. EEG-based methods for human-robot interaction}

Many EEG-based brain-computer interface paradigms present good average performance for communication [1, 8, 18, 10, 9] or HRI [47, 27]. Yet they often require constant operator attention, add additional cognitive burden to the user, require many repeated prompts, and leverage user-specific classification algorithms [50, 36, 31, 34, 48]. Such challenges make these approaches less amenable to real-time control.

ErrPs, however, are naturally occurring brain signals that do not require training or active thought modulation by the human operator [16, 43, 20, 21, 22]. ErrPs have been used in brain-computer interfaces and HRI tasks for performing binary classification, correcting classification errors, and controlling various robot platforms [46, 49, 41, 22, 11, 29, 21, 37, 38. 53. 45]. Nevertheless, most ErrP studies are performed in controlled scenarios because EEG signals have low signalto-noise ratios that make real-time classification challenging [40, 6, 7, 31, 34, 48].

\section{B. EMG-based methods for human-robot interaction}

Surface electromyography is used to measure muscle activity by placing electrodes on the skin over a muscle. Although muscles have complex dynamics, models and controllers can be designed to operate on these signals [52, 19, 33, 39]. Among the common applications of EMG are control of exoskeletons [17, 28, 39, 51, 24, 2, 15, 35], control of prostheses [12, 44], continuous trajectory control [3, 30, 5, 4], and gesture-based control [13, 26]. The presented framework uses a gesture-based EMG control system to actively indicate desired robot behavior. This allows the human to assert direct control over the robot on demand or upon detection of an error.

\section{Hybrid control methods for human-robot interaction}

By combining different biosignal sensors such as EEG, EMG, and eye tracking, researchers have demonstrated promising success controlling prosthetic limbs and even quadrotor flight [42, 25, 23, 32]. This paper focuses on safetycritical tasks where robot operation must be corrected by a human supervisor with low latency. This requires attention to several challenges not jointly tackled in the literature. These include 1) classification of EMG signals on a rolling basis to allow human control at arbitrary and a priori unknown time instances, 2) fast ErrP classification online, 3) a framework for hybrid control management, and 4) experimentally validated reliable system performance in "plug-and-play" settings.

\section{EXPERIMENTAL DESIGN}

An experiment was designed that allows users to supervise and control an autonomous robot solely via brain and muscle activity. The chosen task emulates a safety-critical operation in which a robot moves a power drill to one of three possible targets on a mock plane fuselage. This represents a factory setting where robots assist humans in construction tasks by drilling holes or inserting fasteners.

Two experimental paradigms were implemented: the main closed-loop supervisory control task, and an open-loop session of controlled gestures to acquire additional EMG data. In each one, the user is not required to have prior knowledge or experience controlling a robot or using EEG and EMG sensors. Novice users can thus immediately interact with the robot in ways that are more intuitive than learning a series of button presses, natural language dictionaries, or programming.

\section{A. Main paradigm: closed-loop control, EEG and EMG}

The main task constitutes an autonomous robot performing the target selection task while a human supervises and intervenes when necessary. As shown in Figure 1, the human supervisor wears an EEG cap and EMG electrodes while sitting behind the robot and observing the task. The human mentally judges whether the robot chooses the correct target in each trial, and uses gestures to correct the robot's trajectory when necessary. An EEG classifier detects the presence of ErrP signals upon initial robot motion, and an EMG classifier continuously analyzes muscle activity to identify gestures. 


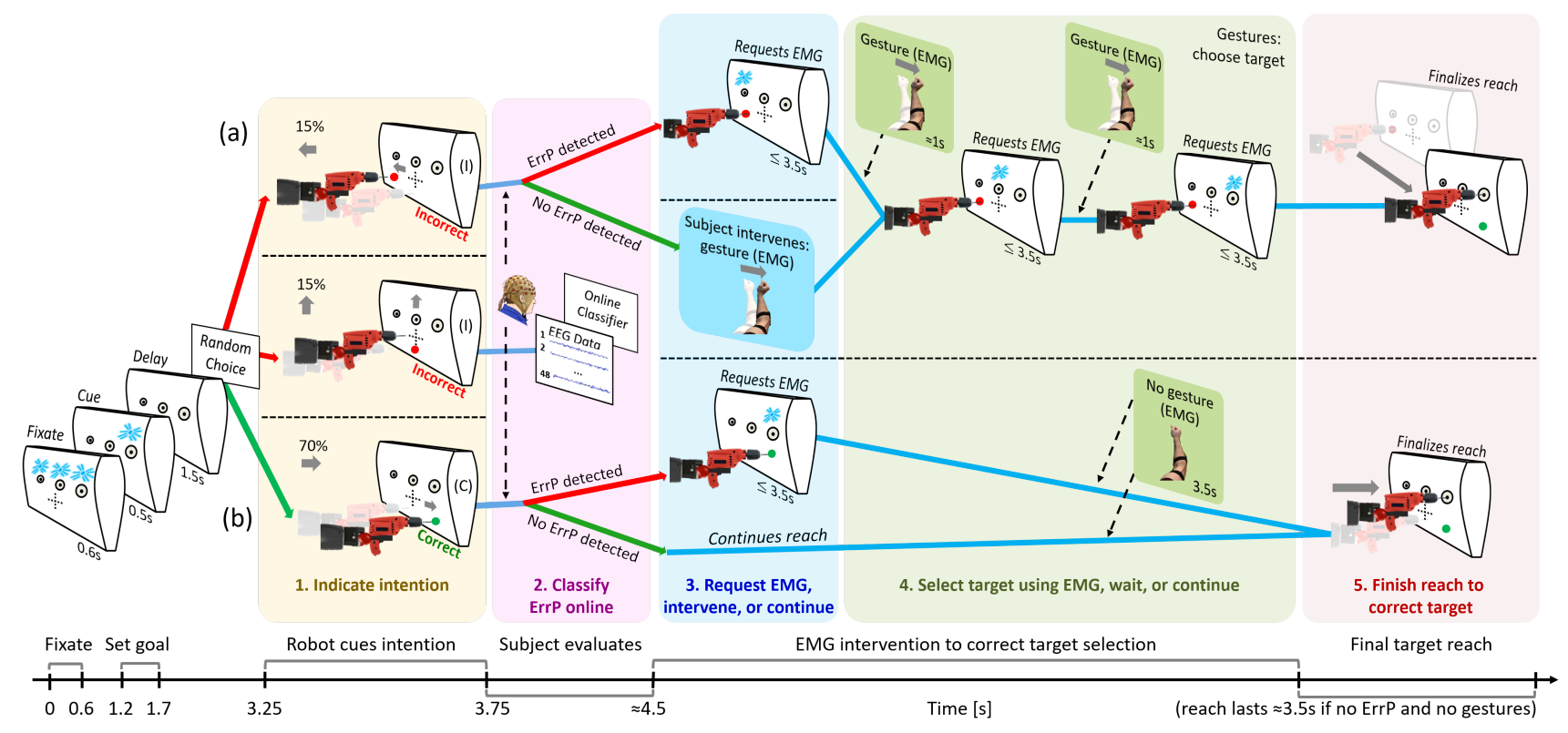

Fig. 2: The sequence of events in the main EEG+EMG paradigm is presented for option (a), when the robot incorrectly chooses the leftmost target, and (b), when the robot correctly chooses the rightmost target. The sequence of events when it incorrectly chooses the center target would be similar to (a). After the correct target is cued, the robot indicates an intention (1-orange block); the user mentally evaluates this choice while ErrP classification is performed (2-purple block). If an ErrP is classified, the robot stops and waits for EMG gestures; otherwise, it continues reaching while searching for any EMG intervention (3-blue block). If a correction is required, the user scrolls through targets via gestures while the robot is stopped (4-green block). Upon target selection or no intervention, the robot completes the reach (5-red block).

Figure 2 illustrates this paradigm and some of its possible progressions in more detail. Three LEDs placed on the mock plane fuselage indicate the left, center, and right targets. For each trial, the system randomly chooses a correct drilling location with uniform probability. All LEDs blink initially to inform the user of the beginning of the trial, then one of the three is turned on for $100 \mathrm{~ms}$ to indicate which location was chosen as correct. Approximately $750 \mathrm{~ms}$ later, the robot randomly chooses a target with a $70 \%$ chance of being correct and then makes an initial arm movement to cue this intention. The user mentally evaluates whether this movement indicates the correct or incorrect target, while a real-time EEG classifier assesses if the user's brain activity presents an ErrP signal.

If an ErrP is found, the system stops the robot, illuminates the LED representing the robot's currently chosen target, and waits for the human to select the correct target using gestures. A gesture can either be a brief flexion (left motion) or extension (right motion) of the right hand. Since the robot's chosen target can be more than one target away from the correct one, several gestures can be performed. Every time a gesture is detected by the EMG classifier, the illuminated LED is changed to indicate the new selection.

If no ErrP signal is found, the robot will continue operating autonomously and reach towards the selected target. However, the user can still interrupt the robot by gesturing left or right at any time; this provides a safety feature in case of inaccurate EEG classification. The robot then stops and waits for correct target selection, which is performed via EMG as described above. The initial intervention gesture also acts to alter the illuminated current selection.
The system considers a target selection finalized if $3.5 \mathrm{~s}$ have elapsed since the last detected gesture. At this point, all LEDs turn off and the robot continues its reach towards the chosen target. If the incorrect target was chosen during the gesture period, the supervisor can again use gestures to interrupt the robot and initiate another target selection period.

Once the robot reaches the selected target, either with or without intervention, it pauses briefly to indicate completion and then returns to its starting position. This concludes a single trial. On average, an experimental session consisted of 4 blocks of 40 trials each and lasted approximately 2 hours.

\section{B. EMG-only paradigm: open-loop gestures}

Since the main paradigm typically produces a relatively small number of gestures performed at arbitrary times, an EMG-only paradigm was also included. This enlarges the corpus of EMG training data and facilitates evaluation of EMG classification performance in a controlled fashion. However, the EMG training data collected during these blocks was not used to train a new classifier for that subject; each experiment used an EMG classifier trained on EMG-only training sessions from previous subjects. Data from EMG-only blocks of three initial subjects that did not participate in online sessions was also included in the training corpus.

For these trials, the subject is positioned in exactly the same manner as described for the main paradigm but the EEG cap is not worn and the robot is not controlled. All three fuselage LEDs blink at the start of each trial to gain the subject's attention, then either the left or right LED illuminates for $0.5 \mathrm{~s}$ to indicate whether a left or right gesture 


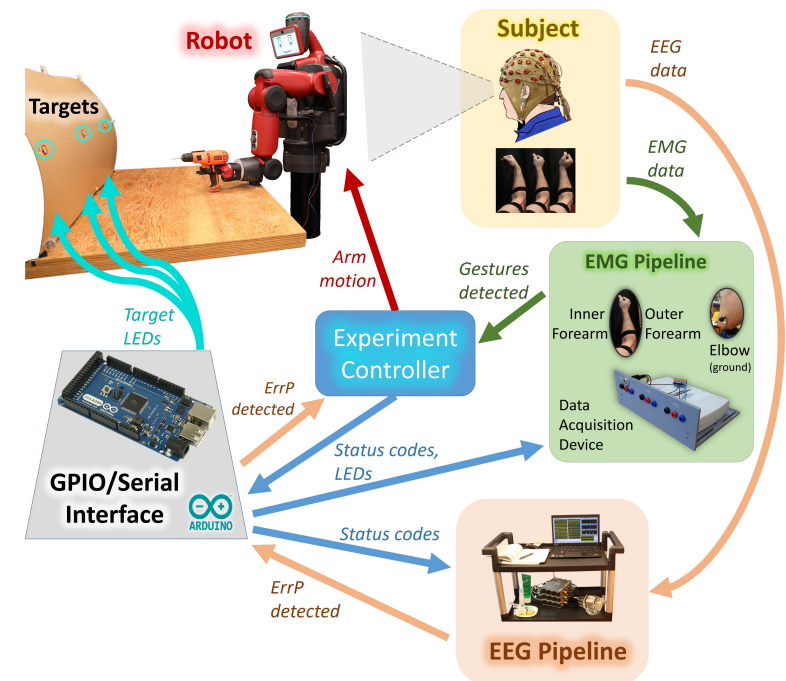

Fig. 3: The system includes an experiment controller and the Baxter robot as well as EMG and EEG data acquisition and classification systems. A mechanical contact switch on the robot's arm detects initiation of robot arm motion. A human supervisor closes the loop.

should be made. After a brief delay, all LEDs illuminate for 1 second; the subject starts and completes their gesture during this period, encouraging consistent gesture timing. The trial therefore implements a "ready-set-go" sequence.

This EMG-only block was performed for new subjects at the beginning of each experimental session. It consisted of 50 trials and lasted approximately 5 minutes.

\section{EEG data paradigm}

To develop a "plug-and-play" system generalizable to any user, the EEG classifier was trained with data from three subjects that were not involved in the online experiments. Unlike the EMG case, there is no EEG-only paradigm; EEG data used for training was obtained from the main closed-loop paradigm. From these previous recordings, correct and incorrect trials were extracted by only selecting the window from $200 \mathrm{~ms}$ to $800 \mathrm{~ms}$ after the robot begins its initial movement. The subjects are instructed to avoid making gestures during this period of ErrP detection, to ensure motor-free EEG activity.

\section{Subject selection}

A total of 7 subjects participated in the online control experiments (71.4\% male, $85.7 \%$ right-handed). No previous experience using EMG, EEG, or brain-computer interfaces was required. Subjects were not screened based on EMG or EEG signals. All subjects provided written consent for the study, which was approved by MIT's Committee on the Use of Humans as Experimental Subjects.

\section{System OVERVIEW AND DATA ACQUisition}

An integrated end-to-end system was developed to enable real-time hybrid supervisory control during the target selection tasks. An overview of the system is shown in Figure 3 . It includes an experiment controller, an EMG subsystem, an EEG subsystem, and the robot. A human supervisor closes the loop.

\section{A. Experiment controller and robot}

The experiment controller, implemented in Python, coordinates all subsystems to realize the paradigms. It chooses correct and selected targets, commands the robot and LEDs, and interprets classifier outputs in the context of the experiment.

For this particular implementation, the Rethink Robotics Baxter robot was used. It communicates via the Robot Operating System (ROS) with the experiment controller, which provides joint angle trajectories for Baxter's left 7 degree-offreedom arm. A physical pushbutton switch is also fastened to the bottom of Baxter's arm to determine exactly when the arm lifts off from the table; this is used as a time-locking signal for the EEG acquisition and classification [41].

An Arduino Mega 2560 serves as an interface for the experiment controller to send status updates to the EMG and EEG subsystems. Key events such as trial timing, chosen targets, robot motion, and LED states are sent to the Arduino via USB serial. These are mapped to predefined 7-bit code words, which the Arduino uses to set a parallel port. The $8^{\text {th }}$ pin of the port is wired to the physical pushbutton switch on Baxter's arm. The EMG and EEG data acquisition systems read this port along with their respective biosignals, allowing for synchronization between data and experimental events.

\section{B. EMG hardware and data acquisition}

Two differential pairs of reusable non-adhesive surface bar electrodes were placed over the user's right posterior (outer) forearm and right anterior (inner) forearm, positioned over the muscles using recommendations in [14]. A single fifth electrode was placed slightly distal to the left elbow as a ground reference. Electrode placement sites were cleaned with Nuprep skin preparation gel and alcohol prep pads to reduce impedance and improve adhesion. Conductive gel was then applied beneath each electrode. A single elastic strap was placed around each forearm to hold the electrodes in place.

The electrodes were connected directly to an NI USB-6216 data acquisition (DAQ) device via a custom breakout board. The parallel port of the Arduino, reflecting experimental event codes, and the LED control signals were also inputs to the DAQ. The 16-bit analog input channels were configured for $\mathrm{a}-0.2 \mathrm{~V}$ to $+0.2 \mathrm{~V}$ range. Differential mode was used to reduce common noise from wireless interference, power line contamination, and motion artifacts. Analog signals were sampled at $2000 \mathrm{~Hz}$, and the samples were sent via USB as buffers of 200 samples every $0.1 \mathrm{~s}$. This data was acquired by Simulink (2017b), which performed online signal processing and gesture identification. Classification results were sent asynchronously to the experiment controller via ROS.

\section{EEG hardware and data acquisition}

A total of 48 passive electrodes, following the 10-20 scalp distribution, were used for EEG data collection. Three Guger Technologies USBamps sampled all signals at $256 \mathrm{~Hz}$. Ground and reference electrodes were placed at the AFz position and the right ear, respectively. The parallel port of the Arduino was also connected directly to GPIO inputs of the USBamps. 


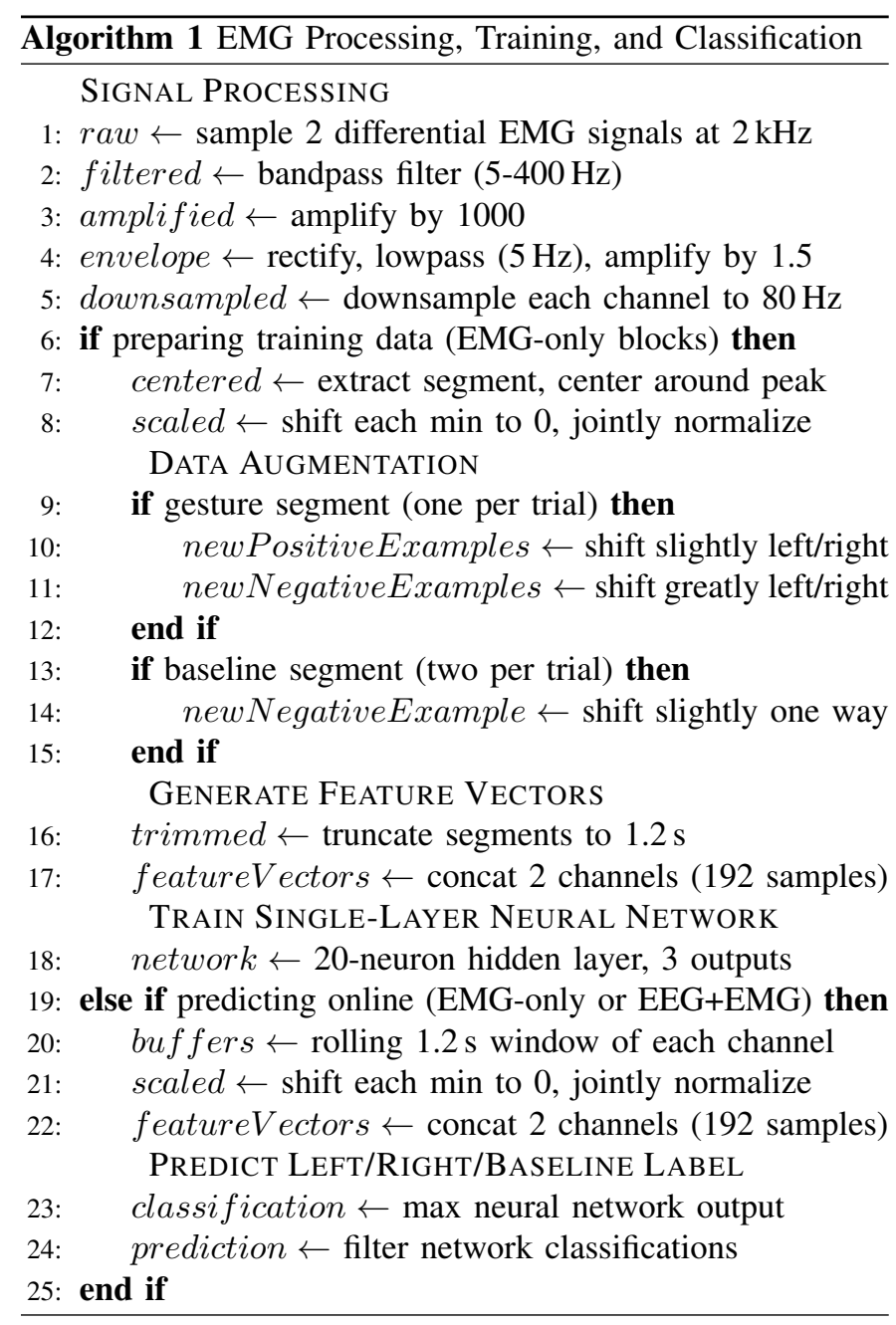

Online signal processing and classification was implemented in Simulink (2015a). For online classification, the switch on Baxter's arm is used to trigger initiation of the EEG buffer that will be passed to the EEG classifier in order to determine whether an ErrP is present. Classification results are sent asynchronously to the experiment controller via the Arduino, using a USBamp GPIO output.

\section{Classification of EMG and EEG Signals}

Two independent classification pipelines were used: one for continuous gesture detection from surface EMG signals and one for time-locked error detection from EEG signals. Neither used training data from each new subject, but rather trained networks based on previously collected data.

\section{A. EMG classification: continuous gesture detection}

As described in Section IV-B, muscle signals are acquired from the inner and outer right forearm. These two signals are then passed through a pipeline of signal processing and classification in order to detect gestures on a rolling basis in real time. The signal processing, feature vector extraction, and classification pipeline is outlined in Algorithm 1

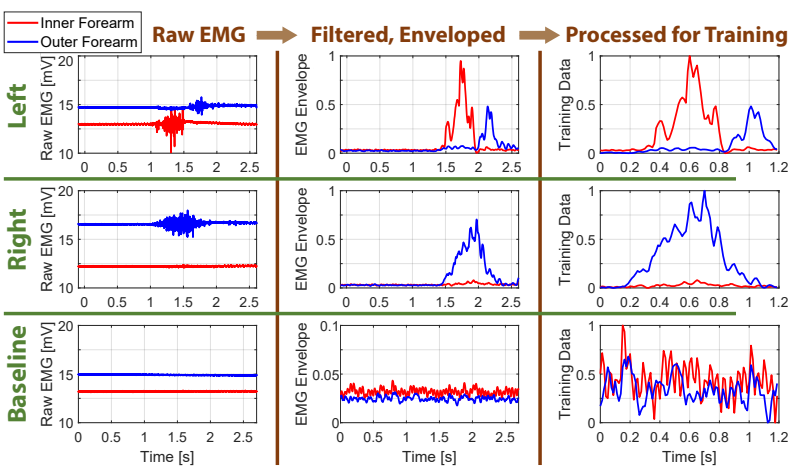

Fig. 4: Acquired EMG signals are passed through a signal-processing pipeline. Raw signals for randomly selected left-gesture, right-gesture, and baseline segments are shown on the left. Detected envelopes are shown in the center. The right column shows the segments after shifting down to 0 , normalizing, trimming, centering, and downsampling.

1) Signal processing: Each muscle signal is independently band-pass filtered, amplified, and envelope-detected. The band-pass filter is an FIR high-pass filter with stopband and passband edge frequencies of $2 \mathrm{~Hz}$ and $5 \mathrm{~Hz}$, respectively, in series with an FIR low-pass filter with passband and stopband edge frequencies of $400 \mathrm{~Hz}$ and $450 \mathrm{~Hz}$, respectively. This preserves the useful frequency content of the EMG signal [14] while removing DC offsets, low-frequency motion artifacts, and high-frequency noise. The filtered signal is amplified by a factor of 1000 . The envelope is then detected by rectifying, applying an FIR $2^{\text {nd }}$-order low-pass filter with passband edge frequency $5 \mathrm{~Hz}$, and amplifying by a 1.5 gain. Examples of raw EMG signals and detected envelopes are shown in Figure 4.

2) Segmentation and normalization: As described in Section III-B, EMG training data was collected by cueing subjects to make left or right gestures during specified time windows using LEDs. The data was then segmented according to those LED signals. To accommodate variable reaction times and gesture durations, each extracted segment begins $0.75 \mathrm{~s}$ before the LEDs turn on and ends $0.75 \mathrm{~s}$ after the LEDs turn off. This produces one labeled gesture segment per trial. Two additional segments were also extracted from each trial when the LEDs were off to represent baseline segments without gestures.

For each segment, each EMG channel's envelope is independently shifted so that its minimum value is at 0 . Both envelopes are then scaled by the same factor such that the peak value becomes 1 . Finally, they are downsampled to $80 \mathrm{~Hz}$. The right column of Figure 4 presents sample results. This shifting and scaling helps normalize inputs across subjects and time, making the network robust to variations in the EMG signal magnitude and offset. Normalizing each segment instead of using a constant scaling factor throughout the experiment helps alleviate issues of calibration, fatigue, and gesture variations.

3) Data augmentation: To continuously detect gestures on a rolling basis, the trained network should be robust to small time shifts while preferring gestures that are centered in the buffer. This helps make predictions smooth and reliable over time while eliminating false detections. For example, the center-top graph of Figure 4 illustrates antagonistic muscle activity during a left gesture that may result in predicting two 


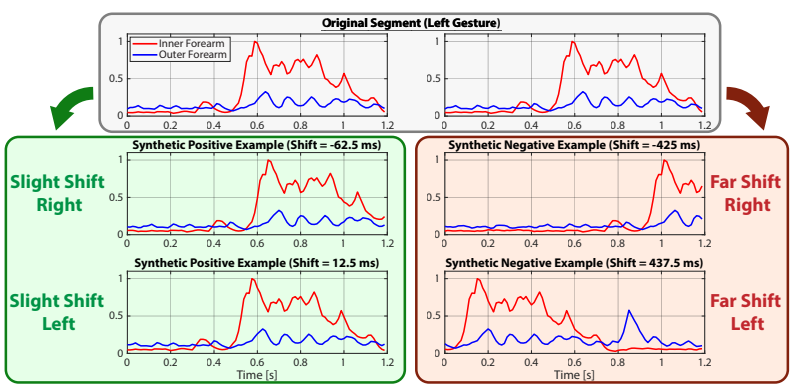

Fig. 5: Training data is augmented by synthesizing new examples from time-shifted copies of original segments. Each gesture trial is shifted slightly left and right to create new positive examples, and shifted farther left and right to create new "baseline" examples.

different gestures, first a left gesture then a right gesture, if the network is too insensitive to time shifts.

A data augmentation approach was used to guide the network towards such robust rolling classification, as shown in Figure 5. For each extracted segment representing a gesture, the data is first centered around the peak value. Two copies are then synthesized by shifting slightly to the left and right by random amounts between 0 and $100 \mathrm{~ms}$; these are assigned the original gesture label. Two copies are also synthesized by shifting farther left and right by $400 \mathrm{~ms}$ plus a random amount up to $100 \mathrm{~ms}$; these are assigned a baseline label. By creating slightly shifted positive examples and significantly shifted negative examples, this data augmentation helps guide the network towards preferring gestures that are centered in the buffer within a specified tolerance.

For each original baseline segment, a single synthetic baseline example is extracted by shifting left or right $400 \mathrm{~ms}$ plus a random amount up to $100 \mathrm{~ms}$. Every segment is then truncated to $1.2 \mathrm{~s}$ (96 samples), using a window centered around the original centered segment. If there is not enough data in the original segment to synthesize one of the shifted examples, then that synthetic example is discarded.

4) Neural network training: As a result of the data augmentation, each trial yields 3 positive gesture examples, 2 baseline examples with shifted gestures, and 4 baseline examples without gestures. The training corpus is thus somewhat biased towards negative examples; this is acceptable since the rollingwindow classifier will encounter vastly more baseline segments than gestures during online experiments, and avoiding false gesture detections is crucial for smooth operation.

The two EMG envelopes within each training example are concatenated to yield a feature vector with 192 elements. These labeled vectors are used to train a feed-forward neural network, using the Pattern Recognition functionality of Matlab's Neural Network Toolbox (version 2017b). The network has a single hidden layer of size 20 using a hyperbolic tangent sigmoid activation function, and an output layer of size 3 using a softmax activation function. The three outputs are used to indicate whether the segment was classified as baseline, a left gesture, or a right gesture.

For each experiment, a new classifier was trained using data from the EMG-only blocks of all previous subjects.
5) Online continuous classification: As data is acquired by Simulink (2017b), the two EMG channels are passed through the signal-processing pipeline described above and then the detected envelopes are downsampled to $80 \mathrm{~Hz}$. These two downsampled envelopes are used to populate rolling buffers of duration $1.2 \mathrm{~s}$. Each time these buffers are updated, the channels are independently shifted down to 0 , jointly normalized, and concatenated. The resulting 192-sample vector is passed to the trained neural network. To avoid spurious predictions that might adversely affect the robot task, raw network classifications are slightly filtered. A rolling buffer of 12 network classifications $(150 \mathrm{~ms})$ is maintained, and a final gesture prediction is declared if at least $60 \%$ of the classifications are not baseline and at least $60 \%$ of the classifications are the same label.

\section{B. EEG classification}

The EEG classification pipeline was trained before the beginning of the online experiments and then remained constant. The same classifier was used for all subjects involved in the online sessions to ensure a realistic "plug-and-play" EEG system and the possibility of faulty classifications so the EMG system could take over the control of the task.

1) Signal processing and feature extraction: During each closed-loop trial, a buffer of EEG data is initiated when the pushbutton switch located under the robot's arm is released. This buffer collects $800 \mathrm{~ms}$ of data from the 48 EEG channels, sampled at $256 \mathrm{~Hz}$. From this buffer, a decoding window in the range $200 \mathrm{~ms}-800 \mathrm{~ms}$ post feedback onset is extracted. These signals are band-pass filtered for $1-10 \mathrm{~Hz}$ using a $4^{\text {th }}$-order zero-phase Butterworth filter. Based on offline analysis, only data from 9 electrodes placed on the mid-line central region, corresponding to the locations $\mathrm{FC} 1, \mathrm{FCz}, \mathrm{FC} 2, \mathrm{C} 1, \mathrm{Cz}, \mathrm{C} 2$, $\mathrm{CP} 1, \mathrm{CPz}$, and $\mathrm{CP} 2$, was selected. These 9 filtered channels are concatenated to create a feature vector for classification, yielding 1386 features per trial.

2) Online ErrP classification: Two feed-forward neural networks were trained and evaluated. The first network realizes a three-layer perceptron (input-100-1). The second network uses a deeper structure with 6 layers (input-100-50-100-10-1). Both networks were designed using Matlab's Neural Network Toolbox (version 2017b), and training was performed by optimizing the networks' parameters with the true labels. To perform binary ErrP classification, a threshold was chosen for each network by minimizing the following cost function: Cost $=\sqrt{(1-\text { sensitivity })^{2}+(1-\text { specificity })^{2}}$.

The offline area under the curve (AUC) metrics for the simpler and deeper neural networks were $70 \%$ and $69 \%$, respectively. Offline analysis averaging the regression outputs from both networks, and using an averaged threshold, increased performance by $3 \%$. Thus, the final classification pipeline implemented in Simulink (2015a) uses both networks; after evaluating each one in parallel on a buffer of EEG data, their outputs are averaged and compared to the averaged threshold. This final output is sent to the experiment controller as the ErrP detection flag. 


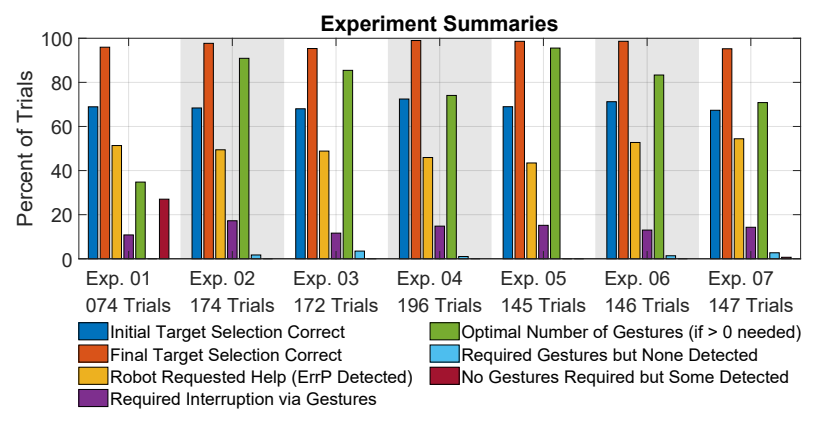

Fig. 6: Overall system performance is summarized by the percent of trials in which the robot placed the drill at the correct target location after full hybrid EEG and EMG control. In addition, the user's interaction and some of the failure modes are described by considering the gestures performed and when they were required.

\section{Results AND Discussion}

The system was used for 7 sessions, each with a different subject. This enabled evaluation of the interface efficacy and of the performance of the EMG and EEG classifiers.

\section{A. System performance: integrated hybrid control}

A summary of results indicating effectiveness of the entire system is shown in Figure 6. There was an average of 156 trials per experiment, after removing trials in which subjects self-reported being distracted. The robot randomly chose the correct target in $69.5 \%$ of the trials, and after EEG and EMG control chose the correct target in $97.3 \%$ of the trials. The hybrid control thus allowed the user to correct errors made by the robot and by the EEG classifier, directing the robot arm towards the correct target. In most cases, the optimal number of gestures required was also detected by the system even though the subjects were not instructed to minimize gestures. The few cases in which the final target was incorrect were typically due to no gesture being detected.

Compared to fully autonomous trials with no error detected via EEG and no gestures detected via EMG, trials in which an error was detected and the user performed gestures to select a new target took on average $8.2 \mathrm{~s}$ longer with standard deviation (SD) $2.8 \mathrm{~s}$. Trials in which no error was detected via EEG but the user interrupted the robot by performing gestures took on average $5.6 \mathrm{~s}$ (SD 2.4 s) longer than fully autonomous trials.

\section{B. EMG classification performance}

The rolling EMG classification was evaluated during the EMG-only blocks as well as during the main paradigm blocks with EMG and EEG. In all cases, the classifiers are used for subjects that were not included in the training data.

Figure 7 illustrates training examples obtained from three subjects during the EMG-only blocks. It reveals overall trends differentiating gestures, but also illustrates the significant variation between subjects and across trials.

1) Open-loop EMG-only blocks: Figure 8 summarizes classification performance. As described in Section III-B, each trial includes a single specified gesture. The classifiers made a single correct gesture prediction in $92.8 \%$ of the 345 trials. There were mixed correct and incorrect gesture predictions in

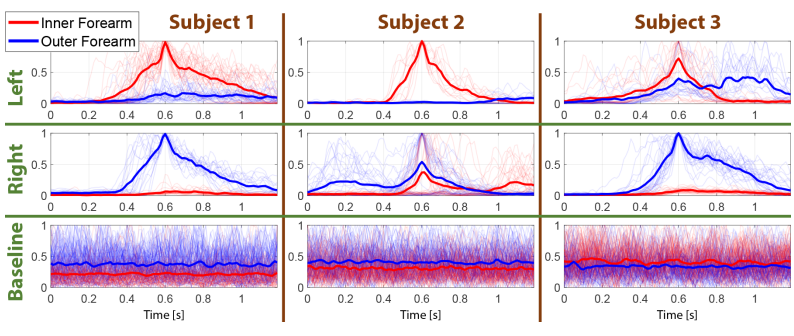

Fig. 7: Training segments extracted from EMG-only blocks are illustrated for three sample subjects. The thin lines show all gestures performed, while the thick lines are average traces. The plotted segments have been vertically shifted, normalized, downsampled, and centered around their peak. Augmentation examples are not included.

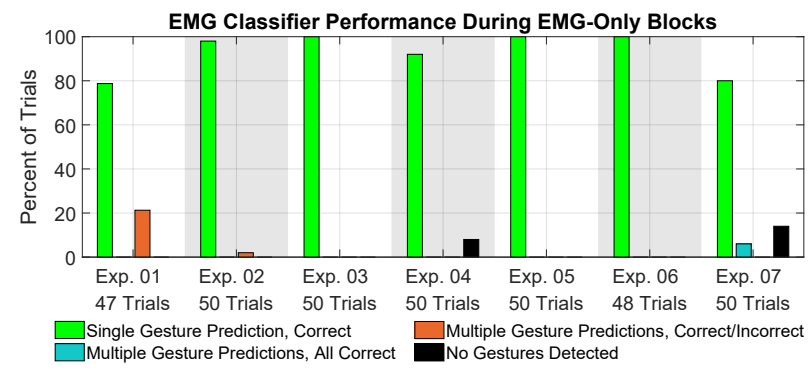

Fig. 8: In each experiment, an EMG classifier trained on previous subjects was continuously invoked during the EMG-only block. The results indicate that the classifiers successfully generalize to new subjects and correctly identify gestures in real time.

$3.2 \%$ of trials, no non-baseline predictions in $3.2 \%$ of trials, and multiple correct-gesture predictions in $0.09 \%$ of trials. There were no trials in which a left or right gesture was classified as the opposite gesture without additional correct predictions. These results indicate that the classifiers robustly and accurately detected gestures during EMG-only blocks.

As described in Section V-A5 the final predictions are filtered versions of the neural network outputs. Without this filter, a single correct gesture prediction would have been made in $77.7 \%$ of trials and multiple correct-gesture predictions would have been made in $15.4 \%$ of trials. This indicates that the filter aided performance by decreasing repeated correct predictions, but was not needed to remove incorrect predictions.

2) Closed-loop EMG+EEG blocks: The EMG classifiers trained on data from EMG-only blocks were also used during the closed-loop trials in which users could arbitrarily make left or right gestures at any time. Ground truth gesture labels for these trials were obtained by annotating videos of the subjects' arms in post-processing. Video was not recorded during the first experiment, so closed-loop performance results for the EMG classifier are not available for that experiment.

Figure 9 and Figure 10 depict the per-experiment and overall confusion matrices for each experiment and across all experiments. The classifiers correctly identified $65.8 \%$ of left gestures and $85.2 \%$ of right gestures, while not falsely identifying any right gestures as left gestures and only $1.8 \%$ of left gestures as right gestures. Most instances in which the classifier missed a gesture altogether occurred when the subject made multiple rapid gestures, such that there were likely multiple gestures within the rolling buffer window. 

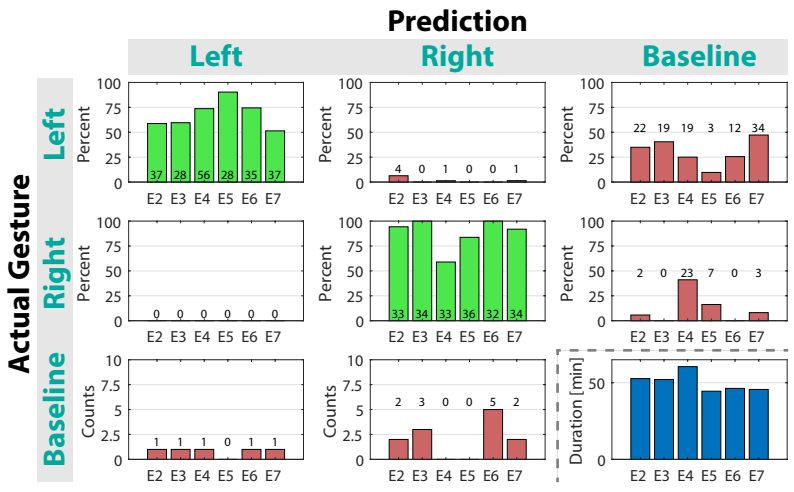

Fig. 9: EMG classification performance during closed-loop EMG+EEG blocks can be summarized by confusion matrices for each experiment. The number on each bar represents the number of instances associated with that percent.

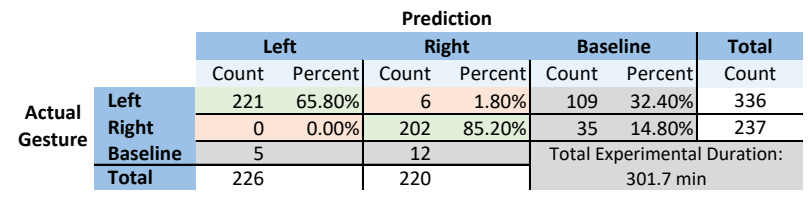

Fig. 10: Summarizing across all experiments, the EMG classification pipeline successfully identified most gestures while avoiding confusing one gesture for another or making spurious predictions.

These results also indicate that the classifiers were generally better at detecting right gestures than left gestures. A possible explanation is that wrist flexion has a higher range of motion than extension, and returning to neutral from flexion often recruits more antagonistic muscles than returning from extension. As seen in Figure 7) the EMG profiles of left flexion gestures show more antagonistic activity and more variability than right gestures for some subjects.

The bottom row of Figure 9 evaluates prediction of baseline activity. In total, the experiments spanned over $18,000 \mathrm{~s}$ and included 17 instances of a gesture being predicted when no gesture was performed. Since the classifiers output predictions at $80 \mathrm{~Hz}$ using a rolling $1.2 \mathrm{~s}$ buffer, this indicates that they performed very well at only detecting gestures when a gesture was actually performed. These metrics evaluate the classifier over the entire span of the experiment, rather than only during the active portions of the trials or when the robot was waiting for gestures. In particular, they include times between trials when the subjects could move and reposition themselves. In light of this, it is promising to see that gestures were very rarely predicted when no gesture was intended by the subject.

The timing of the classification was also analyzed. On average, left-gesture motions lasted for $0.85 \mathrm{~s}$ (SD $0.26 \mathrm{~s}$ ) and were detected by the classifiers $1.15 \mathrm{~s}$ (SD $0.16 \mathrm{~s}$ ) after motion initiation. Right-gesture motions lasted for $0.79 \mathrm{~s}$ (SD $0.23 \mathrm{~s}$ ) and were detected by the classifiers $1.09 \mathrm{~s}$ (SD 0.11 s) after motion initiation. Thus, the classifiers generally waited to predict a gesture until it was contained by and relatively centered in the $1.2 \mathrm{~s}$ window. Once a detection commenced, that prediction was continuously outputted for an average of $0.20 \mathrm{~s}$ or $0.26 \mathrm{~s}$ (both SD $0.08 \mathrm{~s}$ ) for left and right gestures, respectively; the classifiers outputted smooth predictions until

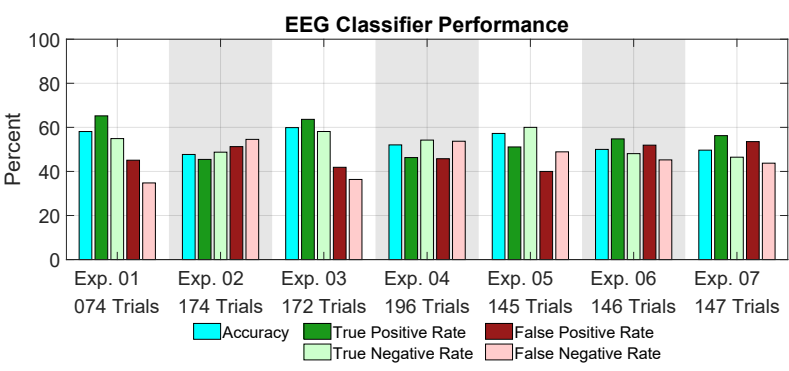

Fig. 11: The EEG classifier performance is summarized by the overall accuracy, true positive rate, and true negative rate in each experiment.

the gesture began moving out of the rolling window. Together, these results imply that the data augmentation successfully encouraged the networks to be robust to small but not large time-shifts and to prefer gestures centered in the buffer.

Overall, these results indicate that the classifiers provided a reliable method of communicating with the robot via gestures. The low false positive rate indicates that the robot rarely stopped unnecessarily. If a gesture was missed or misidentified, the subject could simply make another gesture to correct the target selection. The low latency from completion of a gesture motion to classifier detection is also within reasonable limits for real-time control and closed-loop feedback. Together, these results demonstrate that the system can be used effectively for supervisory control.

\section{EEG classification performance}

Figure 11 summarizes the performance of the EEG classification system in each experiment. Although the overall performance was lower than the performance of the EMG classification system, the EEG system was trained on only 3 subjects and not updated for each new experiment. Its performance was relatively consistent across subjects, and relatively balanced between detection of positive and negative examples. In future experiments, a larger corpus of ErrP data will be used to train a more robust classifier.

\section{CONCLUSION}

Some of the most important tasks for an HRI to accomplish effectively include detection, correction, and prevention of robot errors. This paper demonstrates an end-to-end system that can achieve real-time error correction in a human-robot task by using a hybrid EEG and EMG classification system. This hybrid system can detect human-perceived robot errors and relinquish control to the human via an intuitive musclebased EMG interface. It is also "plug-and-play," attaining high performance even for new users whose data was not part of the training dataset. This work thereby takes a step towards enabling natural human-robot interaction in situations where effective supervision can mean the difference between a dangerous environment and a safe one, or between a costly mistake and a swift intervention.

\section{ACKNOWLEDGMENTS}

This work was funded in part by the Boeing Company, for which the authors express thanks. 


\section{REFERENCES}

[1] Asieh Ahani, Karl Wiegand, Umut Orhan, Murat Akcakaya, Mohammad Moghadamfalahi, Hooman Nezamfar, Rupal Patel, and Deniz Erdogmus. RSVP IconMessenger: icon-based brain-interfaced alternative and augmentative communication. Brain-Computer Interfaces, 1(3-4):192-203, 2014. doi: 10.1080/2326263X. 2014.996066. URL http://dx.doi.org/10.1080/2326263X. 2014.996066.

[2] Di Ao, Rong Song, and JinWu Gao. Movement performance of human-robot cooperation control based on EMG-driven hill-type and proportional models for an ankle power-assist exoskeleton robot. IEEE Transactions on Neural Systems and Rehabilitation Engineering, 25 (8):1125-1134, Aug 2017. ISSN 1534-4320. doi: 10.1109/TNSRE.2016.2583464. URL http://dx.doi.org/ 10.1109/TNSRE.2016.2583464.

[3] Panagiotis K Artemiadis and Kostas J Kyriakopoulos. An EMG-based robot control scheme robust to time-varying EMG signal features. IEEE Transactions on Information Technology in Biomedicine, 14(3):582-588, 2010. ISSN 1089-7771. doi: 10.1109/TITB.2010.2040832. URL http: //dx.doi.org/10.1109/TITB.2010.2040832.

[4] Panagiotis K Artemiadis and Kostas J Kyriakopoulos. EMG-based control of a robot arm using low-dimensional embeddings. IEEE Transactions on Robotics, 26(2):393-398, April 2010. ISSN 1552-3098. doi: 10.1109/TRO.2009.2039378. URL http://dx.doi.org/ 10.1109/TRO.2009.2039378.

[5] Panagiotis K Artemiadis and Kostas J Kyriakopoulos. A switching regime model for the EMG-based control of a robot arm. IEEE Transactions on Systems, Man, and Cybernetics, Part B (Cybernetics), 41(1):53-63, Feb 2011. ISSN 1083-4419. doi: 10.1109/TSMCB.2010.2045120. URL http://dx.doi.org/10.1109/TSMCB.2010.2045120.

[6] Alexandre Barachant and Stephane Bonnet. Channel selection procedure using Riemannian distance for BCI applications. In Neural Engineering (NER), 2011 International IEEE/EMBS Conference, pages 348-351. IEEE, April 2011. doi: 10.1109/NER.2011.5910558. URL http://dx.doi.org/10.1109/NER.2011.5910558.

[7] Alexandre Barachant, Stéphane Bonnet, Marco Congedo, and Christian Jutten. Classification of covariance matrices using a Riemannian-based kernel for BCI applications. Neurocomputing, 112:172-178, 2013. doi: 10.1016/j.neucom.2012.12.039. URL http://dx.doi.org/ 10.1016/j.neucom.2012.12.039

[8] Niels Birbaumer, Nimr Ghanayim, Thilo Hinterberger, Iver Iversen, Boris Kotchoubey, Andrea Kübler, Juri Perelmouter, Edward Taub, and Herta Flor. A spelling device for the paralysed. Nature, 398(6725):297-298, 1999. doi: 10.1038/18581. URL http://dx.doi.org/10. $1038 / 18581$.
[9] Julie Blumberg, Jorn Rickert, Stephan Waldert, Andreas Schulze-Bonhage, Ad Aertsen, and Carsten Mehring. Adaptive classification for brain computer interfaces. In Engineering in Medicine and Biology Society (EMBC), 2007 Annual International Conference of the IEEE, pages 2536-2539. IEEE, Aug 2007. doi: 10.1109/ IEMBS.2007.4352845. URL http://dx.doi.org/10.1109/ IEMBS.2007.4352845

[10] Jonathan S Brumberg, Alfonso Nieto-Castanon, Philip R Kennedy, and Frank H Guenther. Brain-computer interfaces for speech communication. Speech communication, 52(4):367-379, April 2010. ISSN 0167-6393. doi: 10.1016/j.specom.2010.01.001. URL http://dx.doi.org/ 10.1016/j.specom.2010.01.001.

[11] Anna Buttfield, Pierre W Ferrez, and Jd R Millan. Towards a robust BCI: error potentials and online learning. IEEE Transactions on Neural Systems and Rehabilitation Engineering, 14(2):164-168, June 2006. ISSN 15344320. doi: 10.1109/TNSRE.2006.875555. URL http: //dx.doi.org/10.1109/TNSRE.2006.875555.

[12] Jun-Uk Chu, Inhyuk Moon, Yun-Jung Lee, Shin-Ki Kim, and Mu-Seong Mun. A supervised feature-projectionbased real-time EMG pattern recognition for multifunction myoelectric hand control. IEEE/ASME Transactions on Mechatronics, 12(3):282-290, June 2007. ISSN 1083-4435. doi: 10.1109/TMECH.2007.897262. URL http://dx.doi.org/10.1109/TMECH.2007.897262.

[13] Beau Crawford, Kai Miller, Pradeep Shenoy, and Rajesh Rao. Real-time classification of electromyographic signals for robotic control. In Proceedings of AAAI, volume 5, pages 523-528, 2005. ISBN 1-57735-236-x. URL http://aaai.org/Papers/AAAI/2005/AAAI05-082.pdf

[14] Carlo J De Luca. Surface electromyography: Detection and recording. DelSys Incorporated, 2002. URL https:// www.delsys.com/Attachments_pdf/WP_SEMGintro.pdf.

[15] Matthew DiCicco, Lenny Lucas, and Yoky Matsuoka. Comparison of control strategies for an EMG controlled orthotic exoskeleton for the hand. In 2004 IEEE International Conference on Robotics and Automation (ICRA), volume 2, pages 1622-1627. IEEE, 2004. doi: 10.1109/ROBOT.2004.1308056. URL http://dx.doi.org/ 10.1109/ROBOT.2004.1308056.

[16] Michael Falkenstein, Joachim Hohnsbein, Jörg Hoormann, and Ludger Blanke. Effects of crossmodal divided attention on late ERP components. ii. error processing in choice reaction tasks. Electroencephalography and Clinical Neurophysiology, 78(6):447-455, 1991. ISSN 0013-4694. doi: 10.1016/0013-4694(91)90062-9. URL http://dx.doi.org/10.1016/0013-4694(91)90062-9

[17] Ranathunga Arachchilage Ruwan Chandra Gopura, Kazuo Kiguchi, and Yang Li. SUEFUL-7: A 7DOF upper-limb exoskeleton robot with muscle-modeloriented EMG-based control. In 2009 IEEE/RSJ International Conference on Intelligent Robots and Sys- 
tems (IROS), pages 1126-1131. IEEE, 2009. doi: 10.1109/IROS.2009.5353935. URL http://dx.doi.org/10. 1109/IROS.2009.5353935.

[18] Matt Higger, Murat Akcakaya, Hooman Nezamfar, Gerald LaMountain, Umut Orhan, and Deniz Erdogmus. A bayesian framework for intent detection and stimulation selection in SSVEP BCIs. IEEE Signal Processing Letters, 22(6):743-747, June 2015. ISSN 1070-9908. doi: 10.1109/LSP.2014.2368952. URL http://dx.doi.org/ 10.1109/LSP.2014.2368952.

[19] Neville Hogan. Adaptive control of mechanical impedance by coactivation of antagonist muscles. IEEE Transactions on Automatic Control, 29(8):681-690, 1984. ISSN 0018-9286. doi: 10.1109/TAC.1984. 1103644. URL http://dx.doi.org/10.1109/TAC.1984. 1103644

[20] Iñaki Iturrate, Javier M Antelis, Andrea Kubler, and Javier Minguez. A noninvasive brain-actuated wheelchair based on a P300 neurophysiological protocol and automated navigation. IEEE Transactions on Robotics, 25(3): 614-627, June 2009. doi: 10.1109/TRO.2009.2020347. URL http://dx.doi.org/10.1109/TRO.2009.2020347.

[21] Inaki Iturrate, Luis Montesano, and Javier Minguez. Single trial recognition of error-related potentials during observation of robot operation. In Engineering in Medicine and Biology Society (EMBC), 2010 Annual International Conference of the IEEE, pages 4181-4184. IEEE, 2010. doi: 10.1109/IEMBS.2010.5627380. URL http://dx.doi.org/10.1109/IEMBS.2010.5627380.

[22] Iñaki Iturrate, Ricardo Chavarriaga, Luis Montesano, Javier Minguez, and José del R Millán. Teaching brain-machine interfaces as an alternative paradigm to neuroprosthetics control. Scientific Reports, 5:13893, Sep 2015. doi: 10.1038/srep13893. URL http://dx.doi.org/10. 1038/srep13893.

[23] Toshihiro Kawase, Takeshi Sakurada, Yasuharu Koike, and Kenji Kansaku. A hybrid BMI-based exoskeleton for paresis: EMG control for assisting arm movements. Journal of Neural Engineering, 14(1):016015, 2017. doi: 10.1088/1741-2552/aa525f. URL http://dx.doi.org/10. 1088/1741-2552/aa525f.

[24] Kazuo Kiguchi and Yoshiaki Hayashi. An EMG-based control for an upper-limb power-assist exoskeleton robot. IEEE Transactions on Systems, Man, and Cybernetics, Part B (Cybernetics), 42(4):1064-1071, Aug 2012. ISSN 1083-4419. doi: 10.1109/TSMCB.2012.2185843. URL http://dx.doi.org/10.1109/TSMCB.2012.2185843.

[25] Byung Hyung Kim, Minho Kim, and Sungho Jo. Quadcopter flight control using a low-cost hybrid interface with EEG-based classification and eye tracking. Computers in Biology and Medicine, 51:82-92, 2014. ISSN 0010-4825. doi: 10.1016/j.compbiomed.2014.04. 020. URL http://dx.doi.org/10.1016/j.compbiomed.2014. 04.020 .
[26] Jonghwa Kim, Stephan Mastnik, and Elisabeth André. EMG-based hand gesture recognition for realtime biosignal interfacing. In Proceedings of the 13th International Conference on Intelligent User Interfaces, pages 3039. ACM, 2008. ISBN 9781595939876. doi: 10. 1145/1378773.1378778. URL http://dx.doi.org/10.1145/ 1378773.1378778 .

[27] Karl LaFleur, Kaitlin Cassady, Alexander Doud, Kaleb Shades, Eitan Rogin, and Bin He. Quadcopter control in three-dimensional space using a noninvasive motor imagery-based brain-computer interface. Journal of Neural Engineering, 10(4):046003, 2013. doi: 10.1088/ 1741-2560/10/4/046003. URL http://dx.doi.org/10.1088/ 1741-2560/10/4/046003.

[28] T Lenzi, SMM De Rossi, N Vitiello, and MC Carrozza. Proportional EMG control for upper-limb powered exoskeletons. In Engineering in Medicine and Biology Society (EMBC), 2011 Annual International Conference of the IEEE, pages 628-631. IEEE, 2011. doi: 10.1109/ IEMBS.2011.6090139. URL http://dx.doi.org/10.1109/ IEMBS.2011.6090139

[29] Alberto Llera, Marcel AJ van Gerven, Vicenç Gómez, Ole Jensen, and Hilbert $\mathrm{J}$ Kappen. On the use of interaction error potentials for adaptive brain computer interfaces. Neural Networks, 24(10):1120-1127, 2011. ISSN 0893-6080. doi: 10.1016/j.neunet.2011.05.006. URL http://dx.doi.org/10.1016/j.neunet.2011.05.006

[30] Natalia M López, Fernando di Sciascio, Carlos M Soria, and Max E Valentinuzzi. Robust EMG sensing system based on data fusion for myoelectric control of a robotic arm. Biomedical Engineering Online, 8(1):5, 2009. ISSN 1475-925X. doi: 10.1186/1475-925X-8-5. URL http: //dx.doi.org/10.1186/1475-925X-8-5.

[31] Fabien Lotte and Cuntai Guan. Regularizing common spatial patterns to improve BCI designs: unified theory and new algorithms. IEEE Transactions on Biomedical Engineering, 58(2):355-362, Feb 2011. ISSN 00189294. doi: 10.1109/TBME.2010.2082539. URL http: //dx.doi.org/10.1109/TBME.2010.2082539.

[32] Jiaxin Ma, Yu Zhang, Andrzej Cichocki, and Fumitoshi Matsuno. A novel EOG/EEG hybrid human-machine interface adopting eye movements and ERPs: Application to robot control. IEEE Transactions on Biomedical Engineering, 62(3):876-889, 2015. ISSN 0018-9294. doi: 10.1109/TBME.2014.2369483. URL http://dx.doi. org/10.1109/TBME.2014.2369483.

[33] Kurt Manal and Thomas S Buchanan. A one-parameter neural activation to muscle activation model: estimating isometric joint moments from electromyograms. Journal of Biomechanics, 36(8):1197-1202, 2003. doi: 10.1016/ S0021-9290(03)00152-0. URL http://dx.doi.org/10.1016/ S0021-9290(03)00152-0.

[34] Dennis J McFarland, Lynn M McCane, Stephen V David, and Jonathan R Wolpaw. Spatial filter selection 
for EEG-based communication. Electroencephalography and Clinical Neurophysiology, 103(3):386-394, 1997. ISSN 0013-4694. doi: 10.1016/S0013-4694(97)00022-2. URL http://dx.doi.org/10.1016/S0013-4694(97)00022-2.

[35] Marcello Mulas, Michele Folgheraiter, and Giuseppina Gini. An EMG-controlled exoskeleton for hand rehabilitation. In 2005 International Conference on Rehabilitation Robotics (ICORR), pages 371-374. IEEE, 2005. doi: 10.1109/ICORR.2005.1501122. URL http: //dx.doi.org/10.1109/ICORR.2005.1501122.

[36] Gernot Müller-Putz, Robert Leeb, Michael Tangermann, Johannes Höhne, Andrea Kübler, Febo Cincotti, Donatella Mattia, Rüdiger Rupp, Klaus-Robert Müller, and José del R Millán. Towards noninvasive hybrid braincomputer interfaces: framework, practice, clinical application, and beyond. Proceedings of the IEEE, 103(6): 926-943, June 2015. ISSN 0018-9219. doi: 10.1109/ JPROC.2015.2411333. URL http://dx.doi.org/10.1109/ JPROC.2015.2411333.

[37] Xavier Perrin, Ricardo Chavarriaga, Francis Colas, Roland Siegwart, and José del R Millán. Brain-coupled interaction for semi-autonomous navigation of an assistive robot. Robotics and Autonomous Systems, 58 (12):1246-1255, 2010. ISSN 0921-8890. doi: 10.1016/ j.robot.2010.05.010. URL http://dx.doi.org/10.1016/j. robot.2010.05.010

[38] Roziana Ramli, Hamzah Arof, Fatimah Ibrahim, Norrima Mokhtar, and Mohd Yamani Idna Idris. Using finite state machine and a hybrid of EEG signal and EOG artifacts for an asynchronous wheelchair navigation. $E x$ pert Systems with Applications, 42(5):2451-2463, 2015. ISSN 0957-4174. doi: 10.1016/j.eswa.2014.10.052. URL http://dx.doi.org/10.1016/j.eswa.2014.10.052.

[39] João Luiz A.S. Ramos and Marco A. Meggiolaro. Use of surface electromyography for human amplification using an exoskeleton driven by artificial pneumatic muscles. In Biomedical Robotics and Biomechatronics, 2014 IEEE $R A S$ \& EMBS International Conference, pages 585-590. IEEE, 2014. doi: 10.1109/BIOROB.2014.6913841. URL http://dx.doi.org/10.1109/BIOROB.2014.6913841.

[40] Bertrand Rivet, Antoine Souloumiac, Virginie Attina, and Guillaume Gibert. xDAWN algorithm to enhance evoked potentials: application to brain-computer interface. IEEE Transactions on Biomedical Engineering, 56(8):20352043, 2009. ISSN 0018-9294. doi: 10.1109/TBME.2009. 2012869. URL http://dx.doi.org/10.1109/TBME.2009. 2012869.

[41] Andres F Salazar-Gomez, Joseph DelPreto, Stephanie Gil, Frank H Guenther, and Daniela Rus. Correcting robot mistakes in real time using EEG signals. In 2017 IEEE International Conference on Robotics and Automation (ICRA), pages 6570-6577. IEEE, June 2017. doi: 10.1109/ICRA.2017.7989777. URL http://dx.doi. org/10.1109/ICRA.2017.7989777.
[42] Andrea Sarasola-Sanz, Nerea Irastorza-Landa, Eduardo López-Larraz, Carlos Bibián, Florian Helmhold, Doris Broetz, Niels Birbaumer, and Ander RamosMurguialday. A hybrid brain-machine interface based on EEG and EMG activity for the motor rehabilitation of stroke patients. In 2017 International Conference on Rehabilitation Robotics (ICORR), pages 895-900. IEEE, 2017. doi: 10.1109/ICORR.2017.8009362. URL http://dx.doi.org/10.1109/ICORR.2017.8009362

[43] Gerwin Schalk, Jonathan R Wolpaw, Dennis J McFarland, and Gert Pfurtscheller. EEG-based communication: presence of an error potential. Clinical Neurophysiology, 111(12):2138-2144, 2000. ISSN 1388-2457. doi: 10.1016/S1388-2457(00)00457-0. URL http://dx.doi.org/ 10.1016/S1388-2457(00)00457-0

[44] Pradeep Shenoy, Kai J Miller, Beau Crawford, and Rajesh PN Rao. Online electromyographic control of a robotic prosthesis. IEEE Transactions on Biomedical Engineering, 55(3):1128-1135, 2008. ISSN 0018-9294. doi: 10.1109/TBME.2007.909536. URL http://dx.doi. org/10.1109/TBME.2007.909536.

[45] Martin Spüler and Christian Niethammer. Error-related potentials during continuous feedback: using EEG to detect errors of different type and severity. Frontiers in Human Neuroscience, 9:155, 2015. ISSN 1662-5161. doi: 10.3389/fnhum.2015.00155. URL http://dx.doi.org/ 10.3389/fnhum.2015.00155

[46] Martin Spüler, Michael Bensch, Sonja Kleih, Wolfgang Rosenstiel, Martin Bogdan, and Andrea Kübler. Online use of error-related potentials in healthy users and people with severe motor impairment increases performance of a P300-BCI. Clinical Neurophysiology, 123(7):1328-1337, 2012. ISSN 1388-2457. doi: 10.1016/j.clinph.2011. 11.082. URL http://dx.doi.org/10.1016/j.clinph.2011.11. 082 .

[47] Luca Tonin, Tom Carlson, Robert Leeb, and José del R Millán. Brain-controlled telepresence robot by motordisabled people. In Engineering in Medicine and Biology Society (EMBC), 2011 Annual International Conference of the IEEE, pages 4227-4230. IEEE, Aug 2011. doi: 10.1109/IEMBS.2011.6091049. URL http://dx.doi.org/ 10.1109/IEMBS.2011.6091049

[48] Carmen Vidaurre, Claudia Sannelli, Klaus-Robert Müller, and Benjamin Blankertz. Co-adaptive calibration to improve BCI efficiency. Journal of Neural Engineering, 8(2):025009, 2011. doi: 10.1088/1741-2560/8/2/025009. URL http://dx.doi.org/10.1088/1741-2560/8/2/025009.

[49] Jonathan R Wolpaw, Herbert Ramoser, Dennis J McFarland, and Gert Pfurtscheller. EEG-based communication: improved accuracy by response verification. IEEE Transactions on Rehabilitation Engineering, 6(3):326333, 1998. ISSN 1063-6528. doi: 10.1109/86.712231. URL http://dx.doi.org/10.1109/86.712231.

[50] Jonathan R Wolpaw, Niels Birbaumer, Dennis J Mc- 
Farland, Gert Pfurtscheller, and Theresa M Vaughan. Brain-computer interfaces for communication and control. Clinical Neurophysiology, 113(6):767-791, 2002. doi: 10.1016/S1388-2457(02)00057-3. URL http://dx. doi.org/10.1016/S1388-2457(02)00057-3

[51] Yue H Yin, Yuan J Fan, and Li D Xu. EMG and EPPintegrated human-machine interface between the paralyzed and rehabilitation exoskeleton. IEEE Transactions on Information Technology in Biomedicine, 16(4):542549, July 2012. ISSN 1089-7771. doi: 10.1109/TITB. 2011.2178034. URL http://dx.doi.org/10.1109/TITB. 2011.2178034 .
[52] Felix E Zajac. Muscle and tendon: Properties, models, scaling, and application to biomechanics and motor control. Critical reviews in Biomedical Engineering, 17(4): 359-411, 1989. URL http://e.guigon.free.fr/rsc/article/ Zajac89.pdf

[53] Huaijian Zhang, R Chavarriaga, Zahra Khaliliardali, L Gheorghe, Inaki Iturrate, and J d R Millán. EEGbased decoding of error-related brain activity in a realworld driving task. Journal of Neural Engineering, 12 (6):066028, 2015. doi: 10.1088/1741-2560/12/6/066028. URL http://dx.doi.org/10.1088/1741-2560/12/6/066028 